\title{
WhatsApp Messenger para prática educativa com adolescentes escolares na prevenção do uso de substâncias psicoativas
}

WhatsApp Messenger as a tool for educational practice with school adolescents to prevent psychoactive substance use

WhatsApp Messenger para práctica educativa con adolescentes escolares para prevenir el uso de sustancias psicoactivas E-mail: eliseudiasbarbalho@gmail.com

Dayane Gomes dos Santos Alves

\section{Resumo}

O relato de experiência tem por objetivo relatar o uso do WhatsApp Messenger para a prática educativa em saúde com adolescentes escolares. É um estudo de abordagem qualitativa, desenvolvido de julho a setembro de 2020 , no WhatsApp Messenger, com 20 adolescentes de duas escolas de ensino médio de Sobral-Ceará, Brasil. Construíram-se tecnologias educativas em saúde, com intuito de fornecer informações em saúde e fomentar o diálogo com os adolescentes sobre o uso de substâncias psicoativas. Todavia, os adolescentes não participaram dos momentos de intervenção como o esperado. Contudo, o WhatsApp Messenger, pode ser utilizado para fins educativos junto a adolescentes escolares, permitindo o compartilhamento de arquivos (vídeos, fotos, documentos, entre outros) e o diálogo entre os participantes.

Palavras-chave: Adolescentes; Substâncias psicoativas; Tecnologia educativa. 


\begin{abstract}
The experience report aims to report the use of WhatsApp Messenger for educational practice in health with school adolescents. It is a qualitative study, developed from July to September 2020, on WhatsApp Messenger, with 20 adolescents from two high schools in Sobral-Ceará, Brazil. Educational technologies in health were built in order to provide health information and foster dialogue with adolescents about the use of psychoactive substances. However, the adolescents did not participate in the intervention moments as expected. However, WhatsApp Messenger can be used for educational purposes with school teenagers, allowing the sharing of files (videos, photos, documents, among others) and dialogue between participants.
\end{abstract}

Keywords: Adolescent; Substance-related disorders; Educational technology.

\title{
Resumen
}

El informe de experiencia tiene como objetivo informar del uso de WhatsApp Messenger para la práctica educativa en salud con adolescentes escolares. Se trata de un estudio cualitativo, desarrollado de julio a septiembre de 2020, en WhatsApp Messenger, con 20 adolescentes de dos escuelas secundarias en Sobral-Ceará, Brasil. Se construyeron tecnologías educativas en salud con el fin de proporcionar información sanitaria y fomentar el diálogo con los adolescentes sobre el uso de sustancias psicoactivas. Sin embargo, los adolescentes no participaron en los momentos de intervención como se esperaba. Sin embargo, WhatsApp Messenger se puede utilizar con fines educativos con adolescentes escolares, permitiendo compartir archivos (vídeos, fotos, documentos, entre otros) y el diálogo entre los participantes.

Palabras clave: Adolescentes; Sustancias psicoactivas; Tecnología educacional.

\section{Introdução}

A adolescência é um período que compreende a faixa etária de 10 aos 19 anos, quando ocorrem grandes transformações, principalmente corporais e mentais, além de mudanças no modo de se expressar e pensar, o que reflete no convívio com a sociedade. Simultaneamente, há expectativas da sociedade em relação ao futuro estudantil, profissional, à transição do ciclo da vida e puberdade (Coutinho et al., 2017).

Nessa fase, os adolescentes se tornam mais expostos e vulneráveis, pois julgam possuir autonomia suficiente para experimentar novas práticas comportamentais, iniciando, de forma precoce, o consumo de bebidas alcoólicas e tabaco, dentre outras substâncias psicoativas, e a prática de maus hábitos alimentares, aumentando, assim, o risco de desenvolver doenças futuras, como diabetes mellitus tipo 2, enfermidades cardiovasculares e obesidade. Além disso, começam prematuramente a atividade sexual que, por muitas vezes, é desprotegida, podendo ocasionar a contaminação por Infecções Sexualmente Transmissíveis (IST) e gravidez não planejada (Cavalcante et al., 2019).

O consumo de substâncias psicoativas é um fenômeno mundial que tem transcendido à categoria de "problema de saúde". Especificamente quanto à faixa etária, o uso de drogas inicia precocemente, intensificando-se com a idade. Nesse ínterim, percebe-se a necessidade de detectar precocemente o uso de substâncias por adolescentes, sob perspectiva intervencionista, especialmente mediante atividades educativas, abordando os principais comportamentos de risco e, ao mesmo tempo, atuando na redução e prevenção das possíveis complicações ocasionadas pelo uso contínuo (Pinto, 2018).

Assim, as atividades educativas em saúde são de suma de importância, para ampliar o conhecimento dos adolescentes sobre temas pertinentes nessa fase da vida, como o uso de substâncias psicoativas. Os profissionais de saúde têm o desafio de alcançar os adolescentes nos diversos cenários em que estão inseridos, especialmente na escola, frequentada pela maioria dos adolescentes.

Esses desafios foram ampliados diante da Covid-19, que atingiu o Brasil em 2020, classificada pela Organização Mundial de Saúde (OMS), no dia 11 de março de 2020, como doença pandêmica, sendo necessário estabelecer o isolamento social e, consequentemente, a suspensão das aulas presenciais, como medida de reforço ao isolamento social (Oliveira, Lucas \& Iquiapaza, 2020; Bezerra et al., 2020). Assim, os profissionais de saúde tiveram que (re)inventar as práticas em diversos cenários, principalmente aquelas voltadas às ações educativas em saúde. 
Nesse contexto, observou-se incremento no uso da internet que se tornou imprescindível no cotidiano das pessoas, especialmente para interação social (Deslandes \& Coutinho, 2020). Percebe-se que as Tecnologias Digitais de Informação e Comunicação (TDIC) têm se difundido em diferentes áreas de atuações na sociedade, devido à facilidade de acesso e rapidez nas trocas de informações, especialmente nesse período de pandemia da Covid-19. No âmbito da saúde, as TDIC se apresentam como ferramenta potente para o desenvolvimento de intervenções educativas de promoção à saúde, uma vez que possui recursos audiovisuais facilitadores e atrativos, com rapidez na troca de informações (Corrêa \& Rozados, 2017).

Nessa perspectiva, objetivou-se relatar o uso do WhatsApp Messenger para a prática educativa em saúde com adolescentes escolares sobre o uso de substâncias psicoativas na adolescência. Essa mídia social foi escolhida por ser bastante difundida entre os adolescentes e possibilitar o compartilhamento de arquivos, abrangendo grande possibilidade de criatividade de conteúdos dinâmicos e interativos, atraindo a atenção dos adolescentes para os temas abordados, propiciando aprendizagem de forma construtiva, descontraída e transformadora.

$\mathrm{O}$ uso do WhatsApp Messenger foi apontado como atividade interventiva que contribui para resultados comportamentais positivos a curto prazo, uma vez que os hábitos saudáveis podem ser instigados por meio dessa mídia social. Entretanto, ainda é restrito o número de publicações sobre o uso dessa ferramenta para promoção da saúde (Stringhini et al., 2019).

O estudo apresenta aos profissionais de saúde uma possibilidade a mais de desenvolvimento de prática educativa em saúde junto a adolescentes, especialmente nesse período de pandemia da Covid-19, em que as aulas presenciais se encontram suspensas, impossibilitando, assim, o desenvolvimento de atividades educativas presenciais nas escolas.

\section{Metodologia}

Trata-se de estudo descritivo, do tipo relato de experiência, de abordagem qualitativa. Para elaboração desta proposta de estudo, foram utilizadas as técnicas que ajudam a melhorar a escrita de artigos, com o objetivo de organizar as informações coletadas (Pereira et al., 2018). Ademais, o presente artigo foi desenvolvido a partir de intervenção educativa na mídia social WhatsApp Messenger, com intuito de fortalecer a prevenção ao uso de substâncias psicoativas entre alunos do ensino médio regular de escolas públicas.

O cenário do estudo foi o município de Sobral-CE, Brasil, que possui população estimada em 197.663 habitantes, em território 2.122,897 km², segundo informações do Instituto Brasileiro de Geografia e Estatística (IBGE), no ano de 2012.

A experiência foi desenvolvida de julho a setembro de 2020, em duas escolas de ensino médio regular da rede estadual da sede do município de Sobral-CE, selecionadas previamente devido ao conhecimento de maior predominância do uso de substâncias psicoativas.

O estudo foi desenvolvido por integrantes da Liga Interdisciplinar de Saúde do Adolescente - LIPSA, entidade autônoma vinculada à Pró-Reitoria de Extensão e Cultura-PROEX da Universidade Estadual Vale do Acaraú (UVA). A LIPSA foi criada em 2015, por acadêmicos do curso de enfermagem, organizada por um grupo de discentes, sob tutoria de docentes do Centro de Ciências da Saúde (CCS), sendo sua filosofia proporcionar melhor formação humana e profissional aos integrantes. Ademais, a LIPSA tem como finalidade incentivar a atuação ativa de estudantes dos Cursos de Enfermagem e Educação Física da UVA em atividades extensionistas, visando contribuir com a sociedade na melhoria da saúde e qualidade de vida dos adolescentes.

A intervenção educativa on-line foi realizada por equipe interdisciplinar de integrantes da LIPSA, sendo duas docentes (enfermeira e odontóloga) do curso de enfermagem e oito discentes dos cursos de enfermagem e educação física da UVA. 
A intervenção foi realizada em três etapas. A primeira se tratou do planejamento da atividade educativa no WhatsApp Messenger com a temática substâncias psicoativas pelos integrantes da LIPSA (docentes e discentes). Para isso, foi importante a busca de literaturas sobre a temática (artigos científicos, materiais do Ministério da Saúde, dentre outros), no intuito de conhecer a melhor maneira de abordar essa temática on-line junto a adolescentes escolares. A partir dessas leituras, foram elaboradas pelos integrantes da LIPSA diversas tecnologias educativas para serem utilizadas a cada semana na intervenção educativa, como vídeos, infográficos, dentre outros.

A segunda etapa do estudo consistiu em apresentar o projeto e os respectivos objetivos, bem como o planejamento da intervenção educativa aos diretores das duas escolas selecionadas. Esse contato ocorreu por meio do WhatsApp Messenger, pois os integrantes da LIPSA desenvolviam previamente atividades educativas nas referidas escolas, presencialmente, portanto, eram conhecidos da direção das escolas. Após a aprovação dos diretores, estes apresentaram o estudo aos professores e, em seguida, aos alunos do ensino médio que estavam assíduos às aulas on-line e aos responsáveis destes.

Em seguida, para condução da intervenção educativa, criou-se um grupo fechado no WhatsApp Messenger para cada escola participante, sendo enviado aos diretores o convite para acessar o grupo, que foi encaminhado aos alunos, a partir dos seguintes critérios de inclusão: estar cursando o ensino médio nas referidas escolas, ativo às aulas on-line, ter acesso à internet e à rede social WhatsApp e desejar participar do estudo. Trinta alunos aderiram à proposta e acessaram os grupos on-line.

A terceira etapa foi o desenvolvimento da intervenção educativa on-line junto aos adolescentes escolares no WhatsApp Messenger, no período de quatro semanas. Em cada semana, utilizou-se de tecnologia educativa específica, confeccionada previamente pelos integrantes da LIPSA, abordando um conteúdo referente à temática uso de substâncias psicoativas. O propósito era que essas tecnologias fornecessem informações em saúde e fomentassem o diálogo com os adolescentes nos grupos on-line. Após as postagens dessas tecnologias nos grupos, os integrantes da LIPSA lançavam uma pergunta aos adolescentes sobre a temática e iniciavam a discussão sobre a prevenção do uso de substâncias psicoativas, de maneira lúdica, inclusiva e atrativa, incentivando a participação e interação dos adolescentes com os membros da LIPSA.

Adotaram-se como critério de descontinuidade no estudo: sair do grupo do WhatsApp antes do término da intervenção educativa. Consideraram-se participantes do estudo aqueles que permaneceram no grupo do início ao fim da intervenção. Desta forma, concluíram a intervenção educativa vinte alunos das duas escolas selecionadas.

O projeto do estudo foi submetido à Plataforma Brasil, sendo avaliado e aprovado pelo Comitê de Ética em Pesquisa (CEP) da Universidade Estadual Vale do Acaraú, conforme parecer n. 3.896 .393 e Certificado de Apresentação para Apreciação Ética n. 26503819.1.0000.5053. O estudo foi norteado pela Resolução 466/12, do Conselho Nacional da Saúde, que estabelece as Diretrizes e Normas Regulamentadoras de Pesquisas Envolvendo Seres Humanos (Brasil, 2012).

\section{Resultados e Discussão}

A intervenção educativa nos grupos do WhatsApp Messenger teve duração de quatro semanas, com a interação entre os integrantes da LIPSA e os adolescentes escolares, no intuito de ampliar o conhecimento e fortalecer a prevenção do uso de substâncias psicoativas, de maneira lúdica, atrativa e inovadora, em ambiente on-line utilizado pelos adolescentes.

Os adolescentes tiveram uma semana para ingressar nos grupos do WhatsApp Messenger, mediante os convites on-line enviados às escolas. Nesse período, os integrantes da LIPSA realizaram o acolhimento e a apresentação dos integrantes, bem como do projeto e dos objetivos do estudo. Nas três semanas subsequentes, discutiram-se as temáticas sobre o uso de substâncias psicoativas, a partir da utilização de recursos tecnológicos criados pela LIPSA, conforme Quadro 1. 
Quadro 1 - Desenvolvimento da temática "Uso de substâncias psicoativas entre adolescentes", de acordo com a semana, os recursos utilizados e os objetivos. Sobral-CE, 2020.

\begin{tabular}{|c|c|c|c|}
\hline $\begin{array}{l}\text { Semana/ } \\
\text { Período }\end{array}$ & Temáticas & Recursos tecnológicos & Objetivos \\
\hline Semana 1 & Acolhimento dos participantes & Roda de conversa online & $\begin{array}{l}\text { Apresentar os participantes dos } \\
\text { grupos do WhatsApp. } \\
\text { Criar vínculos com os } \\
\text { adolescentes. }\end{array}$ \\
\hline Semana 2 & $\begin{array}{l}\text { Classificação e efeitos no } \\
\text { organismo e fatores de risco } \\
\text { quanto ao uso de drogas na } \\
\text { adolescência }\end{array}$ & Vídeo & $\begin{array}{l}\text { Informar sobre os riscos que o uso } \\
\text { de drogas e os riscos para a saúde, } \\
\text { como também a classificação das } \\
\text { drogas mais utilizadas por essa } \\
\text { faixa etária e a respectiva } \\
\text { incidência. }\end{array}$ \\
\hline Semana 3 & $\begin{array}{l}\text { Fatores de proteção contra o } \\
\text { uso de drogas na adolescência }\end{array}$ & $\begin{array}{l}\text { Infográfico } \\
\text { Vídeo }\end{array}$ & $\begin{array}{l}\text { Informar sobre os fatores que } \\
\text { contribuem para prevenção do uso } \\
\text { de drogas. } \\
\text { Conhecer a rede de apoio de } \\
\text { prevenção e redução de danos ao } \\
\text { uso de substâncias psicoativas. }\end{array}$ \\
\hline Semana 4 & $\begin{array}{l}\text { Experimentação, uso, abuso e } \\
\text { dependência de drogas } \\
\text { É você quem usa? E agora? }\end{array}$ & Card Interativo & $\begin{array}{l}\text { Explicitar os males da } \\
\begin{array}{l}\text { experimentação, uso, abuso e } \\
\text { dependência } \\
\text { psicoativas, de }\end{array} \text { substâncias } \\
\text { consequências na vida dos } \\
\text { indivíduos. }\end{array}$ \\
\hline
\end{tabular}

Fonte: Autores (2020).

Na segunda semana, apresentou-se um vídeo informativo com a temática "Classificação, efeitos no organismo e fatores de risco quanto ao uso de drogas na adolescência”, norteado por publicações do Ministério da Saúde e da Secretaria Nacional de Políticas sobre Drogas (Brasil, 2010; Brasil, 2011). O vídeo foi enviado ao grupo on-line e, em seguida, iniciada discussão sobre o tema.

Foi importante iniciar as discussões sobre o uso de substâncias psicoativas abordando os efeitos no organismo, apresentando para os adolescentes que algumas substâncias são capazes de modificar as funções do Sistema Nervoso Central (SNC), produzindo euforia ou alterando estados de consciência e vigília, as quais ficaram conhecidas como substâncias psicoativas, como ópio, morfina, álcool, cocaína, tabaco (nicotina) e, mais recentemente, alguns compostos sintéticos, como os barbitúricos, os benzodiazepínicos, as anfetaminas, entre outros (Barbosa \& Dalgalarrondo, 2003; Beyer, Staunton \& Moodley, 2014).

Sobre os fatores de risco para o uso de substâncias psicoativas, estudo apontou que não morar com os pais e ter amigos que fazem uso de bebidas foram fatores associados a uma maior probabilidade de uso de bebidas por adolescentes. A 
visão do adolescente quanto ao uso de substâncias psicoativas é influenciada pelo espaço social e geográfico no qual se insere (Almeida \&Felix, 2020).

Outro estudo destacou que dentre os fatores de maior risco para uso de drogas, têm-se o modo de enfrentar os problemas, a falta de apoio religioso e apoio profissional, juntamente com as dificuldades de comunicação dentro das famílias (Cid-Monckton \& Pedrao, 2011).

Na terceira semana, abordou-se a temática "Fatores de proteção contra o uso de drogas na adolescência", a partir da elaboração de um vídeo e um infográfico (Figura 1) que continham informações sobre prevenção ao uso de substâncias psicoativas, de caráter individual, familiar, escolar e social. Optou-se por construir um infográfico, devido à facilidade de acesso dos alunos e por conter informações curtas, porém extremamente úteis para o conhecimento. As informações usadas para essa construção foram obtidas em fontes confiáveis.

Figura 1 - Infográfico confeccionado pela LIPSA sobre prevenção do uso de substâncias psicoativas na adolescência. Sobral-

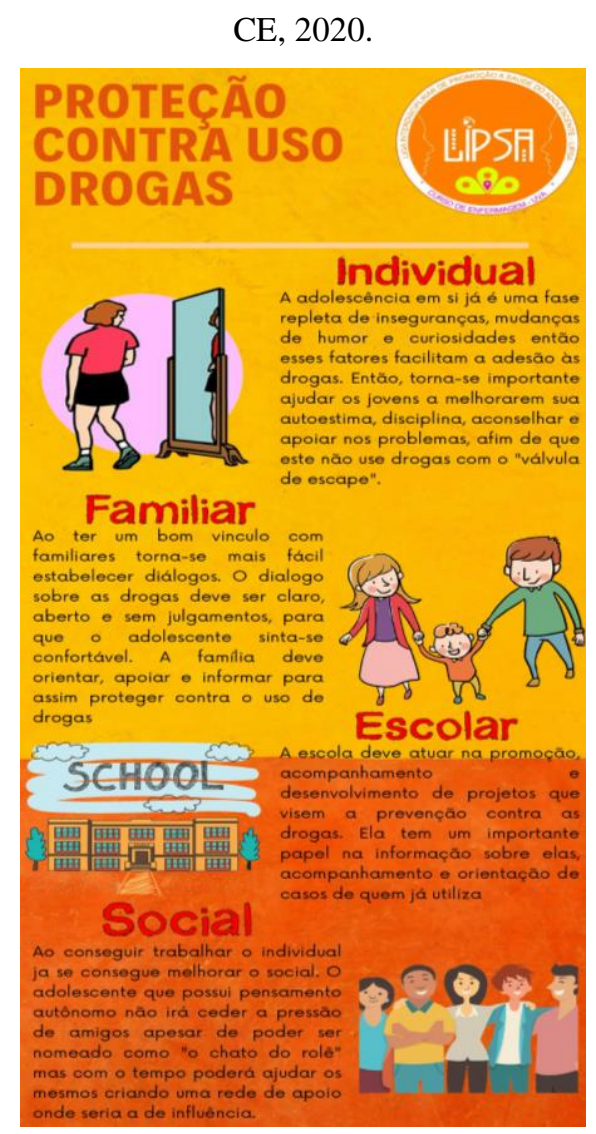

Fonte: Autores (2020)

A Figura 1 acima destacada, mostra o Infográfico utilizado na intervenção para a promoção da saúde dos adolescentes, detalhando o apoio que o adolescente necessita na prevenção do uso e no enfrentamento do consumo de substâncias psicoativas. A aplicação das TDIC torna a comunicação com os jovens mais dinâmica e criativa. Ademais, conforme citado, o infográfico demonstra para o adolescente que é fundamental para saúde: o apoio individual, elevando a autoestima e gerando a auto aceitação; a família, incentivando o diálogo com os pais no sentido de orientar sobre as substâncias psicoativas; a escola, contribuindo significativamente através de projetos que abordam essa temática para os jovens; o apoio social de colegas, amigos e conhecidos, gerando influências positivas a partir de exemplos de vida.

Para essa temática também foi produzido um vídeo que foi compartilhado nos grupos on-line, com objetivo de informar a rede de apoio à prevenção ao uso de drogas e a redução de danos, apresentando opções para os adolescentes. 
A probabilidade de experimentação de álcool e cigarro aumenta a partir dos 15 anos, a idade e a prática religiosa, por sua vez, atuam como fatores de proteção à experimentação do álcool (Andrade et al., 2017). Dentre os fatores de proteção, o núcleo familiar e os valores repassados pela família constituem fatores que podem propiciar, ou não, o uso abusivo de substâncias psicoativas pelo adolescente (Rosa, 2017).

Existe a necessidade de intervir em relação ao uso de drogas entre adolescentes em idades precoces, pois o uso e abuso dessas substâncias ocorrem cada vez mais cedo, sendo preciso que os adolescentes saibam das reais consequências e grandes chances de dependência (Zeitoune et al., 2012). Na quarta semana, a atividade teve como tema "Experimentação, uso, abuso e dependência de drogas. É você quem usa? E agora?". Utilizou-se de cards interativos, com ilustrações que despertassem a atenção dos adolescentes, cujo objetivo era apresentar, de maneira criativa, os males da experimentação, o uso e abuso de substâncias psicoativas, evidenciando as consequências na vida dos adolescentes.

As tecnologias em saúde contribuem para o aprendizado, como uma cartilha que foi utilizada para prevenção do uso de drogas de abuso entre os adolescentes, pois, de maneira didática e dinâmica, mostrou-se um instrumento válido e confiável para ser utilizado na promoção da saúde e prevenção de drogas entre adolescentes (Moura et al., 2019).

As redes sociais, por agregar diversas pessoas, contribuem para formação de grupos, constituem também ambientes propícios para aprendizagem em saúde. Desse modo, estudo evidenciou que a mídia social facebook pode ser utilizada com os adolescentes para a prática educativa em saúde (Aragão et al., 2018).

As ações educativas propostas foram relevantes para prevenção do uso de substâncias psicoativas na adolescência, visto que foi notória a praticidade de disseminação das informações, sendo simples o processo de confecção dos recursos tecnológicos e o compartilhamento nos grupos do WhatsApp.

Percebeu-se que após as postagens das tecnologias confeccionadas pela LIPSA (vídeos, infográficos, cards, dentre outros), os adolescentes visualizavam as postagens, mas mesmo com as perguntas lançadas pelos integrantes da LIPSA, poucos comentavam ou compartilhavam experiências. Acredita-se que esse fato se deve ao uso de substâncias psicoativas que, muitas vezes, é encoberto, não sendo fácil para os adolescentes discorrerem abertamente sobre esse assunto. Outro motivo para a pouca participação pode ser o fato de os adolescentes não conhecerem pessoalmente os integrantes da LIPSA, já que a primeira interação foi on-line, para a intervenção educativa.

A despeito da pouca participação dos adolescentes, considera-se que foi importante discutir a temática referente ao uso de substâncias psicoativas com esses sujeitos, para que possam ter conhecimento dos efeitos e das consequências que essas substâncias podem trazer para saúde. Ademais, considera-se preocupante a porcentagem de professores que vivenciam alunos fazendo uso de drogas nas dependências da escola. Os professores possuem dificuldades formativas, institucionais, culturais e pedagógicas que podem prejudicar a abordagem dessa temática em sala de aula (Sandim et al., 2020). Por isso, a relevância da prática educativa em saúde junto a adolescentes escolares.

\section{Considerações Finais}

O estudo apontou que o aplicativo WhatsApp Messenger pode ser utilizado com intuito educativo junto a adolescentes escolares, considerando que é utilizado entre esse público, permitindo o compartilhamento de arquivos (vídeos, fotos, documentos, entre outros) e o diálogo entre os participantes. O uso do WhatsApp para discutir a temática relativa às substâncias psicoativas foi possível, após o planejamento e a pactuação com a direção das escolas, os pais e os adolescentes.

Durante a pandemia da Covid-19, foi imprescindível a utilização dessas TDIC para interagir com os adolescentes escolares, realizando atividades educativas em saúde, necessárias nessa fase da vida, como o uso de substâncias psicoativas. Mesmo com as aulas presenciais suspensas, em respeito ao isolamento social, como medida de prevenção ao novo coronavírus, 
os adolescentes tiveram a oportunidade de obter informações em saúde, interagir com acadêmicos de enfermagem e de educação física, além de diminuir dúvidas sobre o assunto em foco.

Para tal fim educativo, é necessário o planejamento prévio, de modo criativo e interativo, para facilitar a aprendizagem. Os recursos tecnológicos utilizados (vídeos, cards, infográficos) produzidos pela LIPSA intentaram compartilhar informações de maneira criativa, atrativa e clara.

Os profissionais de saúde têm papel indispensável na prática educativa em saúde junto a adolescentes, de modo a potencializar o diálogo e a interação entre os jovens. Para os integrantes da LIPSA, a experiência foi de grande aprendizado, o que possibilitou maior interação entre os acadêmicos de enfermagem e de educação física e os adolescentes escolares.

Como limitações, menciona-se a insuficiente participação dos adolescentes na intervenção on-line, muitos visualizavam as publicações nos grupos, mas poucos comentavam e/ou compartilhavam experiências.

Em função da grande relevância da temática, recomenda-se para trabalhos futuros a realização de estudos com maior amplitude abordando a utilização das TDIC com fins educativos com adolescentes; fiéis utilizadores da internet e das mídias sociais. Salienta-se também a pertinência de se investigar as motivações dos adolescentes para interação; o que desperta o desejo de expor ideias e sentimentos entre grupos.

\section{Referências}

de Almeida, C. S., \& Lana, F. C. F. (2020). Relação entre espaço sociocultural e o consumo de substâncias psicoativas por adolescentes. Revista Gaúcha de Enfermagem, 41 .

de Andrade, M. E., Santos, I. H. F., de Souza, A. A. M., Silva, A. C. S., dos Santos Leite, T., da Cunha Oliveira, C. C., \& de Albuquerque Júnior, R. L. C. (2017). Experimentação de substâncias psicoativas por estudantes de escolas públicas. Revista de saude publica, 51, 1-9.

Aragão, J. M. N., Gubert, F. D. A., Torres, R. A. M., Silva, A. S. R. D., \& Vieira, N. F. C. (2018). The use of Facebook in health education: perceptions of adolescent students. Revista brasileira de enfermagem, 71(2), 265-271.

Barbosa, P. C. R., \& Dalgalarrondo, P. (2003). O uso ritual de um alucinógeno no contexto urbano: estados alterados de consciência e efeitos em curto prazo induzidos pela primeira experiência com a ayahuasca. Jornal Brasileiro de Psiquiatria, 52(3), 181-190.

Beyer, C., Staunton, C., \& Moodley, K. (2014). The implications of Methylphenidate use by healthy medical students and doctors in South Africa. BMC medical ethics, 15(1), 20.

Aquino, E. M., Silveira, I. H., Pescarini, J. M., Aquino, R., \& Souza-Filho, J. A. D. (2020). Social distancing measures to control the COVID-19 pandemic: potential impacts and challenges in Brazil. Ciência \& Saúde Coletiva, 25, 2423-2446.

Brasil. (2012). Conselho Nacional de Saúde. Resolução n 466, de 12 de dezembro de 2012. Aprova normas regulamentadoras de pesquisas envolvendo seres humanos.

Brasil. (2010). Ministério da Saúde. Secretaria de Vigilância em Saúde Departamento de DST, Aids e Hepatites Virais. Álcool e outras Drogas Brasília

Brasil. (2011). Ministério da Justiça. Secretaria Nacional de Políticas sobre Drogas (SENAD). Drogas: cartilha sobre maconha, cocaína e inalantes (2a ed.). Brasília.

Cavalcante, F. M. L., de Sousa, F. W. M., Oliveira, I. K. M., Amaral, H. R. M., Rosa, B. S. C., da Silva Gomes, J., \& Vasconcelos, M. I. O. (2019). Atividades de extensão universitária: um olhar para a promoção da saúde do adolescente. Saúde em Redes, 5(3), 305-315.

Matos Coutinho, B. L., Alves Feitosa, A., Cross Diniz, C. B., Souza Ramos, J. L., Zuqui Ribeiro, L., Rodrigues Amorim, S., \& Pinheiro Bezerra, I. M. (2017). Álcool e drogas na adolescência: processo de trabalho no programa saúde na escola. Revista Brasileira de Crescimento e Desenvolvimento Humano, 27(1).

de Vargas Corrêa, M., \& Rozados, H. B. F. (2017). A netnografia como método de pesquisa em Ciência da Informação. Encontros Bibli: revista eletrônica de biblioteconomia e ciência da informação, 22(49), 1-18.

Cid-Monckton, P., \& Pedrão, L. J. (2011). Factores familiares protectores y de riesgo relacionados al consumo de drogas en adolescentes. Revista LatinoAmericana de Enfermagem, 19(SPE), 738-745.

Deslandes, S. F., \& Coutinho, T. (2020). O uso intensivo da internet por crianças e adolescentes no contexto da COVID-19 e os riscos para violências autoinflingidas. Ciência \& Saúde Coletiva, 25, 2479-2486.

Moura, M. I. L. D., Leal, J. B., Leal, J. B., Correia, V. G. A., Leal, J. B. P., Silva, M. G. D., \& Oliveira, A. K. S. D. (2019). Cartilha sobre prevenção do uso de drogas para adolescentes. Rev. enferm. UFPE on line, 1106-1114.

Oliveira, A. C. D., Lucas, T. C., \& Iquiapaza, R. A. (2020). What has the covid-19 pandemic taught us about adopting preventive measures? Texto \& contextoenfermagem, 29. 
Research, Society and Development, v. 10, n. 1, e38110111722, 2021

(CC BY 4.0) | ISSN 2525-3409 | DOI: http://dx.doi.org/10.33448/rsd-v10i1.11722

Pereira, A. S., Shitsuka, D. M., Parreira, F. J., \& Shitsuka, R. (2018). Metodologia da pesquisa científica.

Pinto, A. C. S. (2018). Construção e validação de curso on-line para prevenção do uso indevido de drogas por adolescentes.

Rosa, B., \& Junior, G. A. (2017). A constituição dos vínculos e estilos familiares quando os filhos fazem uso das drogas. Psicologia e Saúde em debate, 3(1), $127-140$.

Stringhini, M. L. F., Chagas, J. D. S., Reis, M. J. M. D., Brito, P. R. T. D., \& Souza, D. S. D. (2019). Whatsapp® como ferramenta de promoção da saúde com diabetes: relato de experiência.

Sandim, L. S., Silva, S. M., Araújo, B. M. D., Santos, P. M. R. D., Naves, E. F., \& Matos, M. A. D. (2020). Drogas entre adolescentes e adultos jovens: estudo com professores de um assentamento do Brasil Central. Nursing (Säo Paulo), 4318-4323.

Zeitoune, R. C. G., Ferreira, V. D. S., Silveira, H. S. D., Domingos, A. M., \& Maia, A. C. (2012). El conocimiento de los adolescentes sobre drogas lícitas e ilícitas: una contribución para la enfermería comunitaria. Escola Anna Nery, 16(1), 57-63. 